\title{
Development and validation of a model for the optimization of regenerative braking of high speed trains
}

\author{
Luca Pugi, Amedeo Frilli, Daniele Nocciolini, Enrico Meli, Andrea Rindi \\ DIEF, Department of Industrial Engineering \\ University of Florence \\ Via Santa Marta 3, 50139 Florence, Italy \\ luca.pugi@unifi.it, amedeo.frilli@unifi.it,daniele.nocciolini@unifi.it,enrico.meli@unifi.it, andrea.rindi@unifi.it
}

\begin{abstract}
The interest for the energetic optimization of railway systems is constantly increasing due to recent developments concerning Technological Standards and Regulations. A particular attention is usually paid to the coupling between the dynamical behaviour of railway vehicles and the electrical infrastructure, including all the subsystems related to energy distribution and storage. In this research work, the authors propose a newly developed modelling approach for the analysis of the railway vehicle-line system, based on the use of the object-oriented language Simscape that introduces significant advantages with respect to other conventional simulation tools in terms of computational efficiency and modularity. In order to validate the modelling approach, the Italian DC High Speed line, considering a high-speed train with distributed traction power based on the new ETR1000, has been used as a benchmark. The proposed model provides accurate results with a high computational efficiency, proving to be an important tool for the analysis of railway systems. This tool has then be used to perform a preliminary energetic optimization of the considered system.
\end{abstract}

Keywords-railway longitudinal dynamics; traction and braking systems; mechatronics; energy storage; regenerative braking.

\section{INTRODUCTION}

Global Warming and the public attention to energetic problems worldwide are producing an increasing interest in the efficiency of transportation systems: in fact, the transportation field is still responsible for a large percentage of the $\mathrm{CO}_{2}$ emissions in Europe and in most of the industrial countries worldwide [1], [2]. In this scenario, railway is still the most efficient transport method from the energetic and environmental points of view but its competitors are continuously improving their performances and hence the challenge to improve the energetic efficiency has become very important even in this field.

Furthermore, the optimization of railways energetic consumptions can bring advantages not only from the environmental point of view but also from the economic one, considering also the possibility to increase the diffusion of High Speed trains. Figure 1 shows an important factor which is strongly pushing towards the energy optimization of railways:

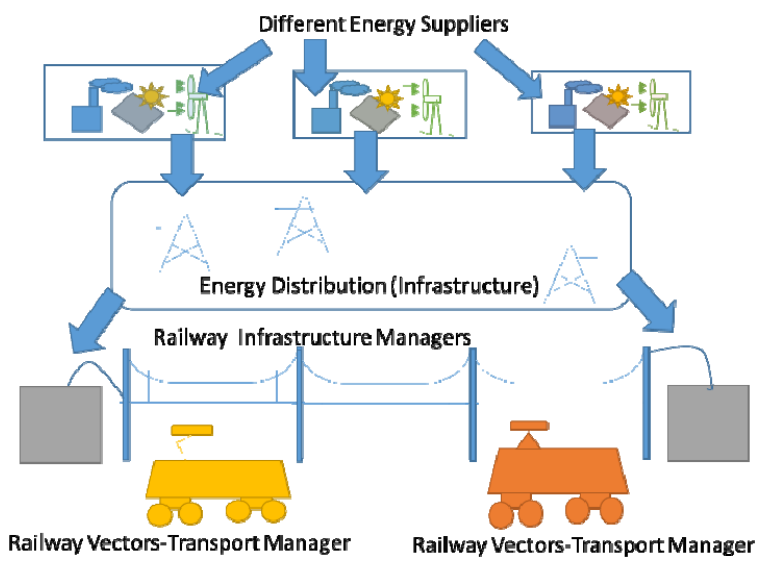

Figure 1: Interactions between the different subjects involved in the railway system.

the ever growing liberalization process of energy and transportation markets is stimulating all the involved stake holders (i.e. the energy infrastructure, the energy supplier the railway infrastructure and the railway vector) to accurately analyse, through simulations and measurements, the energy fluxes involved in the system and their cost.

This complex energetic-economic scenario yields the possibility to differentiate the cost of railway vehicle according to their energetic efficiency. Furthermore, the possibility to acquire energy from different suppliers will allow the optimization of costs based on a number of external factors, e.g. the geographic location of the line.

The interoperability and the competitions arising from this liberalized market [3] will further stimulate the need for a strong energy optimization of the railway system, including all the aspects of this transport field, from the traction and braking systems to the entire electric line.

Another interesting aspect from the energetic point of view characterizes modern railways: the diffusion of distributed traction systems in fact widens the possibility to recover energy during the braking phase. The braking energy recovery allows to drastically reduce the wear of the pneumatic brakes, 


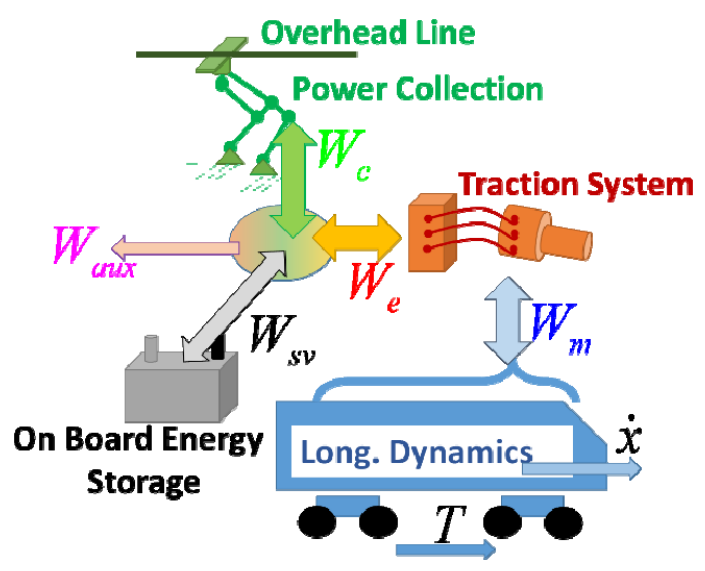

Figure 2: Power balance between different on-board subsystems.

with important advantages both in terms of cost of design and maintenance but also in terms of environmental pollution [4], [5], [6]; this recovered energy can be used during the traction phases in different ways depending on the specific railway application.

The possibility to implement regenerative braking in a railway system depends primarily on the availability of a stationary or on board storage device.

Both types of storage devices have advantages and disadvantages, ranging from costs and encumbrances to the possibility to assure the system autonomy.

Tramways and light rail systems present evident advantages from the use of regenerative braking: thus, energy recovery has been implemented in real systems and widely investigated in many research works found in literature that analyse different aspects of railways energy recovery systems [7-13].

In this research work, the authors investigate the application of regenerative braking in high-speed railways: this railway field presents bigger difficulties in the implementation of energy recovery systems, due to the higher level of energy involved and to the limited number of braking phases. Due to those aspects, an accurate analysis of the entire vehicle-line-storage devices system is mandatory in order to understand which energy recovery strategy could better suit the considered railway system.

A complete railway system is quite complex and includes many different elements and physical phenomena. In order to develop a model able to accurately reproduce the global energetic behaviour of this system, at least two fundamental phenomena must be taken into account: the dynamical behaviour of the train and the electrical response of the line. The first one can be analysed with a mechanical model able to reproduce the longitudinal dynamics of the vehicle and the power needed by the vehicle itself to advance. The other phenomenon can be analysed with an electrical model that includes the behaviour of the contact line and its couplings with the previous model; in order then to analyse the influence of energy storage devices, another submodel representing those devices must be included within the line model.

The proposed model has been developed using the innovative object orient language Matlab-Simscape. This language allows overcoming the limitations of simulation model developed using only Matlab-Simulink [14], [15], like the need to introduce redundant integrated states in order to preserve the modularity of the model. Matlab-Simscape allows the user to describe a component using a physics based approach in terms of balance equations according to the Bond-Graph approach [16].

A Simscape model allows the optimization of the number of integrated states and, thanks to a bidirectional correspondence between the model topology and the physical system, allows creating modular models that can be easily used to simulate a great number of different scenarios. Furthermore, the numerical efficiency of this tool is high, allowing to perform parametric analyses (needed to optimize a system) with limited computational times.

The proposed model is composed of two main submodels: the vehicle longitudinal dynamics model and the contact line model, which includes the submodels of the stationary or on board energy storage devices. The dynamical submodels is coupled with the electrical one through the vehicle position and requested power: those quantities allows the calculation of the voltage and current throughout the considered line. In the following Sections, the main features of those sub models will be exposed.

The final Section shows then the comparison between the results obtained with the proposed model and a set of experimental results and an analysis of the feasibility of energy storage systems within a high speed line: the numerical results are in good agreement with the experimental data and the computational efficiency of the model allows to easily investigate complex and various operating scenarios.

\section{VeChiCle DyNAMiCAL MODEL}

In the mechanical submodel, a position based velocity profile can be imposed to the vehicle through a nonlinear pilot that controls the application of traction and braking efforts.

The efforts applied to the vehicle depends on the train speed and are calculated according to tabulated relationships that depend on the considered train performances. Those traction and braking efforts are exerted on the train through a first order filter in order to correctly take into account the real delay used for the application of traction and braking forces to enhance comfort and avoid extreme mechanical solicitations. Furthermore, those efforts are limited by the value of the wheel-rail adhesion coefficient (see Figure 3) according to Equations (1) and (2):

$$
\begin{aligned}
& T_{T} \leq T_{T \max }=k_{m} m g \mu, \\
& T_{B} \geq T_{B \max }=-m g \mu,
\end{aligned}
$$

where the coefficient $k_{m}$ is defined as the ratio between the vertical load on motorized axis and the total weight of the train. The value of the wheel-rail adhesion can be calculated taking into account TSI (acronym of Technical Specifications for Interoperability) limits [3] or according to Muller model: 


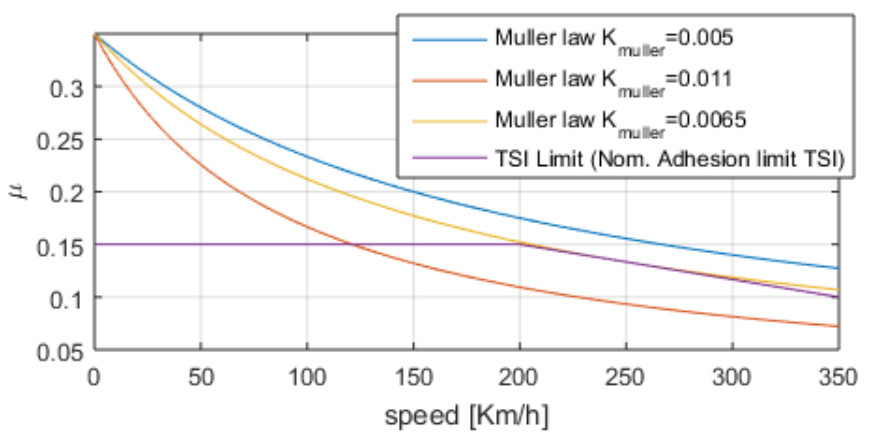

Figure 3: Wheel rail adhesion dependency as a function of the vehicle speed: comparison between Muller model and TSI limits.

$$
\mu=\frac{\mu_{0}}{1+K_{\text {muller }} \dot{x}_{k m h}},
$$

where $\mu_{0}$ is the static friction factor, $\dot{x}_{k m h}$ is the vehicle speed and $K_{\text {muller }}$ is a scaling parameter whose values are between 0.005 and 0.011 .

The analysis of the braking phase includes the possibility to choose different braking effort percentage and above all the possibility to choose a specific blending policy between the electrical and the pneumatic braking systems; the braking power of the two braking systems is then calculated as a function of speed, line voltage and electrification standard and braking state (i.e. service or emergency).

The model includes two efficiency parameters: an electrical efficiency that depends on current and frequency and a mechanical efficiency that depends on the vehicle speed.

The total longitudinal efforts applied to the vehicle and hence the train acceleration can be calculated taking into account the traction and braking efforts and the resistance force due to the slope of the line $\alpha$, to the curves with radius $r(d(r))$ and to the motion resistances calculated according to the coefficients $a, b, c$. Thus, the equation of motion of the vehicle can be expressed as follows:

$m_{i} \ddot{x}=T-m g \sin \alpha-m\left(a \dot{x}^{2}+b \dot{x}+c+d(r)\right)$.

From the longitudinal effort and the train speed, it is possible to calculate the mechanical power required by the vehicle to the traction system as follows:

$$
W_{m}=T \dot{x} \text {. }
$$

During the braking phase, this power can mechanically dissipated by the pneumatic brakes or converted in electrical power by the traction system; the electrical part can be then dissipated on on-board resistors or used to perform some sort of energy recovery. The latter part can be then transferred to the line or stored on on-board devices. Taking into account also the power consumption of auxiliary systems, the complete power balance can be expressed as follows:

$W_{e}=W_{a u x}+W_{s v}+W_{c}$,

where $W_{e}$ is the electrical power due to the traction system, $W_{\text {aux }}$ is the power consumption due to auxiliary systems, $W_{s}$ is

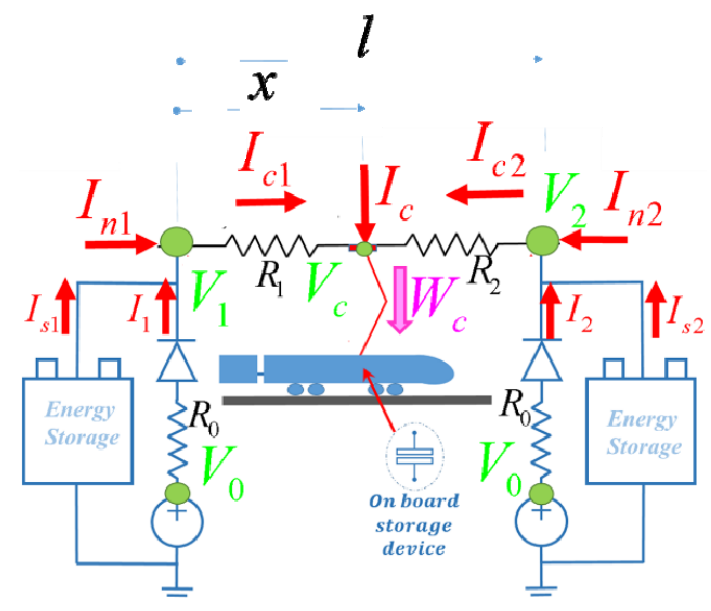

Figure 4: Simplified model of the contact line including the Energy Storage Systems.

the power stored on on-board devices and $W_{c}$ is the power transferred to the line.

The blending strategy (i.e. the management strategy between different braking systems) chosen for this research work is to maximize $W_{e}$ in normal operating conditions.

\section{Contact Line And Sub Stations}

The electrical line model is shown in Figure 4: the electrical substations have been modelled as real voltage generators that can be connected in parallel with the model of a stationary energy storage device. The catenary has been modelled like a variable resistance that depends on the vehicle position; the resistance of the two sections can be calculated as follows:

$$
\begin{aligned}
& R_{1}=\rho x, \\
& R_{2}=\rho(l-x),
\end{aligned}
$$

where $\rho$ is the equivalent linear resistivity of the contact line and $x$ is the position of the train with respect to the distance $l$ between two adjacent ESSs. The stationary energy storage devices that can be included in the analysis are batteries and supercapacitors; the authors have chosen to represent both kind of systems using the same modelling approach. The proposed energy storage device model is a load connected to the substsation (in parallel) which includes an ideal voltage generator and two resistance, respectively in series and parallel with the generator in order to simulate power loss and transient behaviour. This simplified approach allows simulating the complete system behaviour requiring very few input parameters (which can be quite difficult to be found) and allowing preserving a great computational efficiency. If an on board storage device is included in the analyzed physical system, its submodel can be added to the line, connecting it in correspondence of the pantograph and considering, as inputs for the submodel, the line voltage, the state of charge and the power available from the vehicle braking. The no load voltage of the storage system can be calculated as a function of current and State Of Charge; the S.O.C. can be defined as follows: 


$$
\text { S.O.C. }=\int \frac{\eta_{I} \mathrm{I}_{s i}}{E_{\max }} d t,
$$

where $\eta_{I}$ is the efficiency of the device and $E_{\max }$ is the maximum stored energy. The difference between batteries and supercapacitors lays in the different dependency of the device voltage on the S.O.C.. The Bond-Graph approach followed to develop this model presents great advantages in terms of modularity and computational efficiency with respect to previous modelling approaches based on the use of Simulink; furthermore, the proposed model is more suitable for the use on a RTOS target.

\section{Model VAlidation And Results}

The proposed model has been validated through a set of experimental data concerning a traction manoeuvre carried out by the ETR1000 high speed train on the Firenze-Roma line, from standstill to a speed equal to $250 \mathrm{~km} / \mathrm{h}$ applying the maximum traction effort allowed by the train performances.

A comparison between the experimental velocity profile and that calculated with the proposed model is reported in Figure 5: the two velocity profiles are quite similar, highlighting how the parameters used for the model (e.g. train performances and inertia) are correct. The biggest errors on the numerical velocity profile can be detected below $110 \mathrm{~km} / \mathrm{h}$, where the measured performances are higher. This could be due to the real adhesion conditions and to axles skidding phenomena: in fact, during the experimental tests, some skidding phenomena were detected between 80 and $120 \mathrm{~km} / \mathrm{h}$. The difference between the two velocity profiles is the due to the lower continuous guaranteed performance considered in the proposed model: a more accurate modelling of adhesion and skidding phenomena could then allow to further improving the accuracy of the proposed model. Figure 6 and Figure 7 show further comparisons related to the same traction manoeuvre: Figure 6 shows the experimental and numerical power consumption while Figure 7 shows the correspondent voltage in correspondence of the train pantograph. The results concerning the power consumption starts with an initial phase in which the train is stopped and the power requested to the line is due to on board auxiliary systems (e.g. lights and air conditioning). The numerical and experimental results are quite near, with the only exception of the phase where the axle skidding phenomenon occurs.

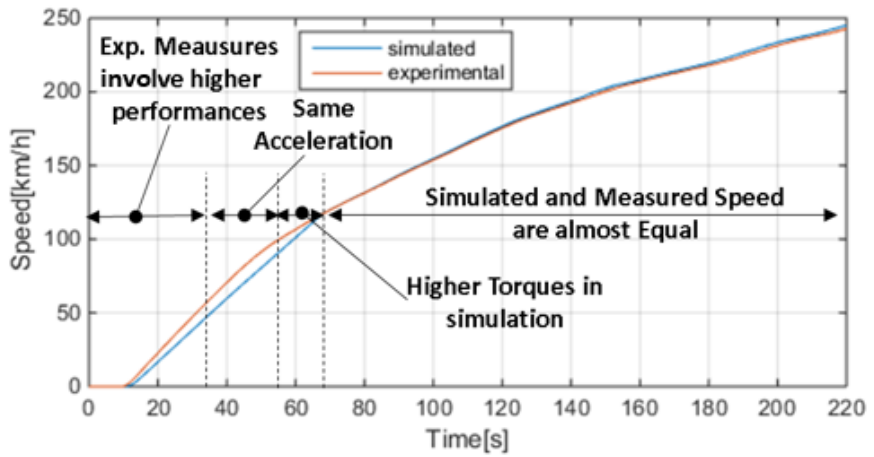

Figure 5: Comparison between an experimental traction manoeuvre and the corresponding numerical results.

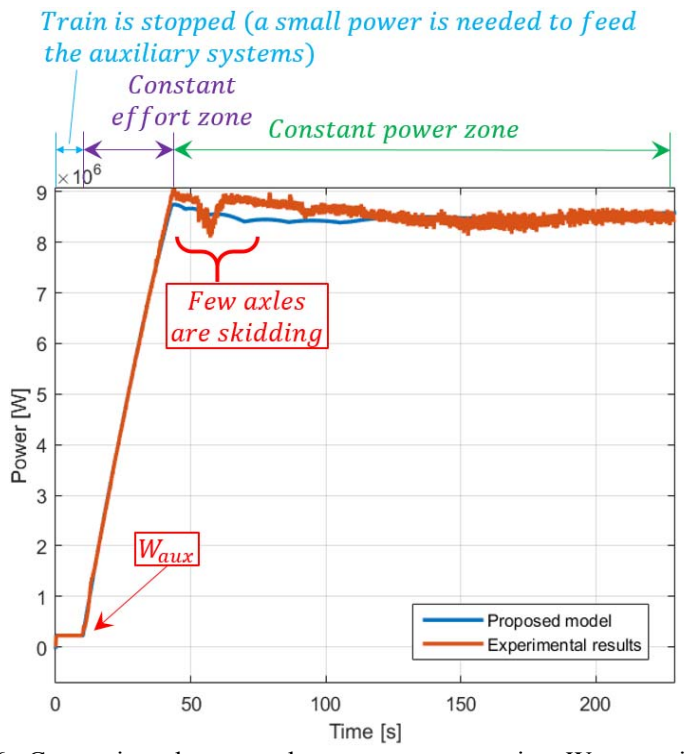

Figure 6: Comparison between the power consumption Wc experimentally measured and the corresponding numerical values.

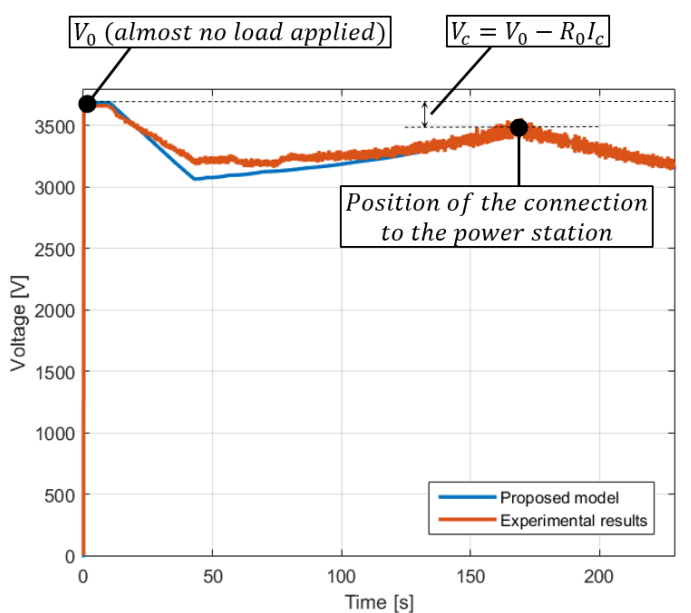

Figure 7: Comparison between the experimental line voltage $\mathrm{Vc}$ (at the pantograph) and the corresponding numerical.

Analogous considerations are valid for Figure 7; the voltage results allows highlighting how the voltage decreases during the traction phase while the train is leaving the first substation. There is then a voltage peak in correspondence of the second substation, where it is possible to verify the value of the substation equivalent impedance used in the proposed model, according to the following equation:

$V_{c}=V_{0}-R_{0} I_{c} \Rightarrow R_{0}=\frac{\left(V_{0}-V_{c}\right)}{I_{c}}$.

After the validation of the proposed model, the authors used the developed simulation tool to carry out a series of simulations with the aim of understanding the feasibility of the use of stationary energy storage devices in high speed trains (the use of on board devices has not been considered due to the characteristics of both the high speed system and the storage device). The model has been simulated considering the ETR1000 on the Firenze-Roma "Direttissima" line; the considered manoeuvre includes a first acceleration with the 


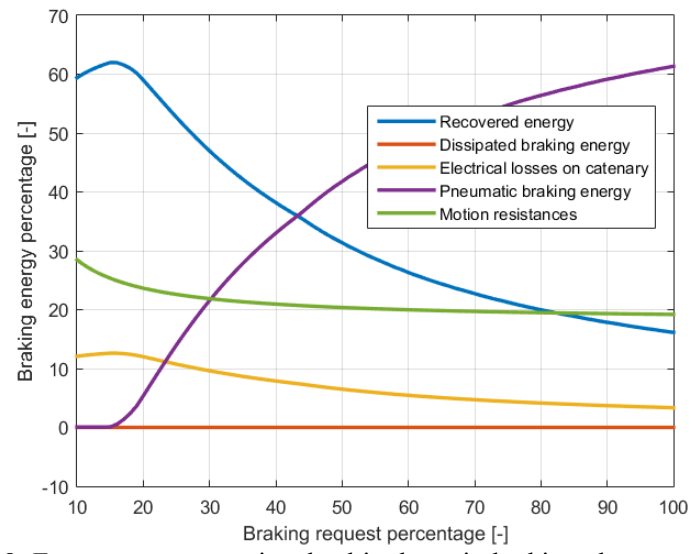

Figure 8: Energy percentages involved in the train-braking phase as a function of the braking request (no voltage limitations).

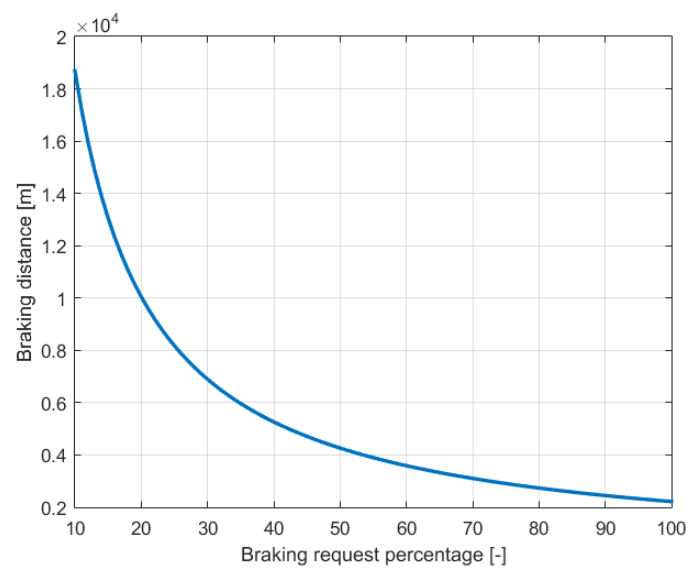

Figure 9: Braking distance as a function of the braking request (no voltage limitations)

maximum traction effort from standstill to $70 \mathrm{~m} / \mathrm{s}$, a long coasting phase and a final braking. The chosen blending strategy is to maximize the electrical power and the maximum braking power is about $5 \mathrm{MW}$.

Figure 8, Figure 9 and Figure 10 show the main results of this set of simulations, all performed considering a braking phase that begins in correspondence of the midspan of two adjacent substations. The results shown in those Figures are reported as functions of the braking request, i.e. considering different percentages of the maximum braking effort. The variation of the braking effort has an important influence on the recovered braking energy: Figure 8 shows how the recovered energy has a maximum in correspondence of a $20 \%$ braking request, when it is possible to perform an electrical braking without the need to add any percentage of pneumatic braking (i.e. with higher requests it is hard to avoid the use of pneumatic braking). However, a $20 \%$ braking request implies huge braking distance and time (see Figure 9 and Figure 10), hence it could be better to choose, as a favourable operating condition for energy recovery, a braking request of about $50 \%$, where the recovered energy is still appreciable but the braking distance and time are acceptable. This first analysis does not consider any limitations on the braking voltage; hence, the energy dissipated on on-board resistors is constantly $0 \%$.

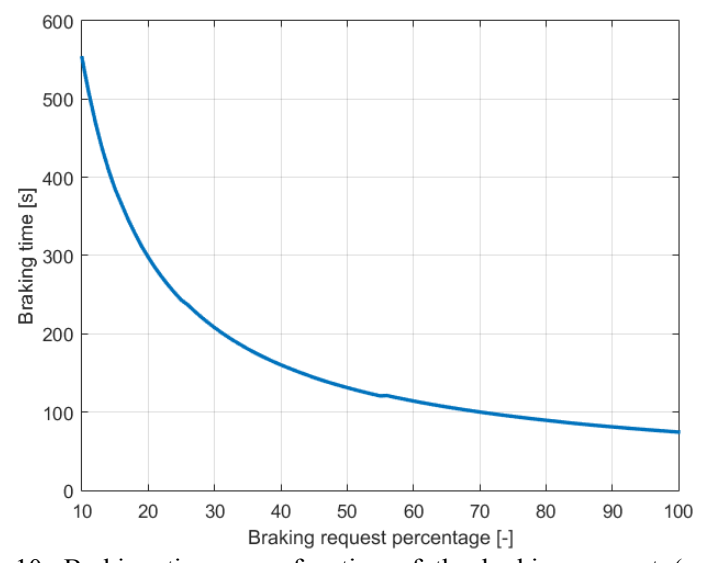

Figure 10: Braking time as a function of the braking request (no voltage limitations).

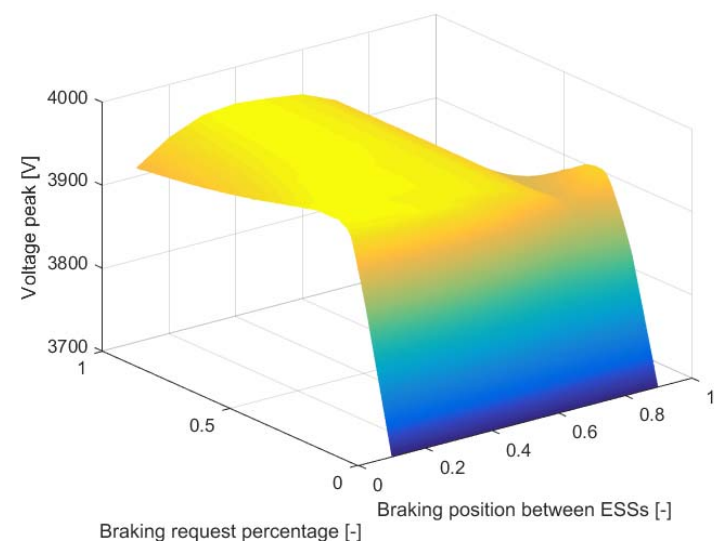

Figure 11: Braking voltage peak as a function of the braking position and of the braking request (no voltage limitations).

Figure 11 shows the influence of the braking position on the braking voltage peak: while the voltage peak is almost constant beyond a $20 \%$ braking request, the distance from the substations at the beginning of the manoeuvre has a bigger importance. In fact, if the braking phase begins near a substation, the braking voltage peak is significantly lower.

This analysis has been repeated considering a voltage limitations: in fact, real trains often uses limitation devices that disconnect the braking energy feeding to the line if the voltage exceeds certain values. In those cases, the surplus electrical braking energy is dissipated on on-board resistors. The voltage limit considered in this analysis is $3900 \mathrm{~V}$ : Figure 12 and Figure 13 show the results obtained in this refined analysis. The energy fluxes reported in Figure 12 are essentially the same as in Figure 8; however, a small energy percentage is now dissipated on on-board resistors.

Figure 13 shows then how the voltage peak map is now almost completely saturated to the limit value (even if the values reported in Figure 11 were not significantly higher): the voltage is below the limit only in close proximity of the substations. Finally, the proposed model is characterized by a high computational efficiency: the simulation of the complete line (about $250 \mathrm{~km}$ ) requires 5 seconds of simulation time. 


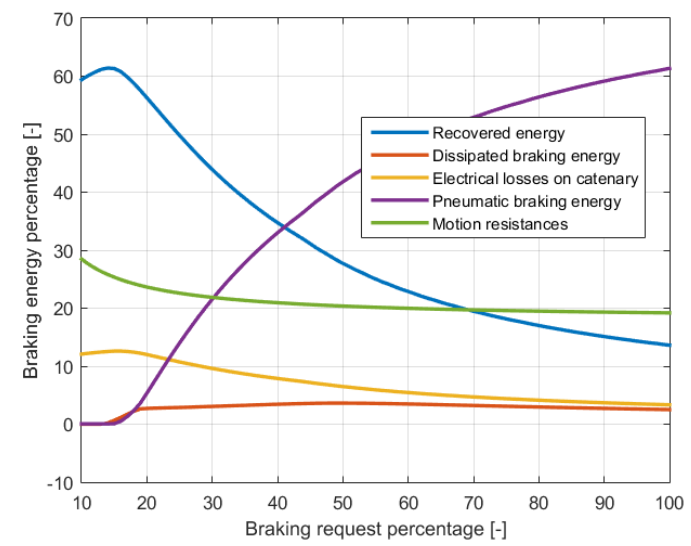

Figure 12: Energy percentages involved in the train braking phase as a function of the braking request (voltage limited to $3900 \mathrm{~V}$ ).

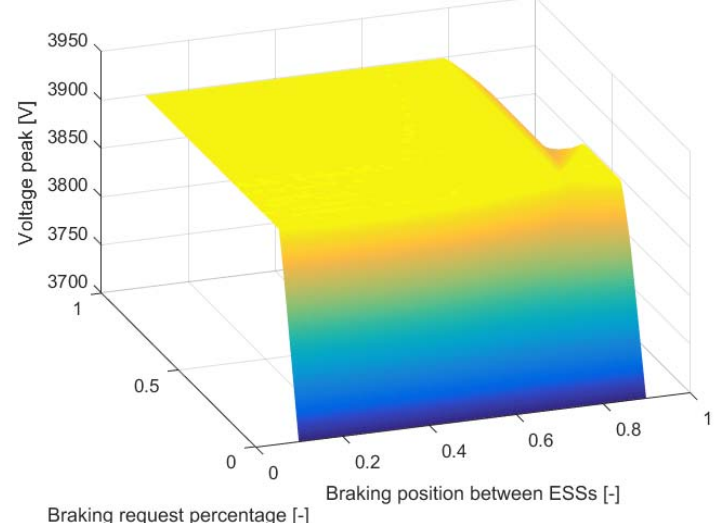

Figure 13: Braking voltage peak as a function of the braking position and of the braking request (voltage limited to $3900 \mathrm{~V}$ ).

\section{CONCLuSIONS AND Future DeVElopments}

The growing interest towards environment and pollution represent a significant boost for the transportation sector in order to reach higher energetic efficiency. For the railway sector, one important way to achieve these results is the recovery of the vehicle energy dissipated during the braking phases, typically using energy storage devices. In this research work, the authors propose an innovative-coupled model for the analysis of the interactions between the train dynamical behaviour and the electrical behaviour of line, substations and storage devices. The model, developed using the innovative object oriented Simscape language, has been validated considering a set of experimental data referred to the highspeed train ETR1000. It has then been used for the feasibility analysis of energy storage devices within a high-speed line: the simulations performed with the proposed model have highlighted that stationary storage devices could allow significant savings even in a high-speed system, where energy storage solutions have typically been discarded. The research work will continue with a further validation of the proposed model and with the development of a more complex energy storage device model, in order to get a better insight on the energy recovery possibilities for high-speed trains.

\section{REFERENCES}

[1] European Environment Office, tech. reports at www.eea.europa.eu

[2] Association of American Railroads tech reports at https://www.aar.org/

[3] TSI, technical specifications for interoperability available at the official site of ERA (European Railway Agency) http://www.era.europa.eu/Core-Activities/Interoperability/Pages/ TechnicalSpecifications.aspx

[4] Abbasi, S., Jansson, A., Olander, L., Olofsson, U. and Sellgren, U., 2012. A pin-on-disc study of the rate of airborne wear particle emissions from railway braking materials. Wear, 284, pp.18-29.

[5] Gehrig, R., Hill, M., Lienemann, P., Zwicky, C.N., Bukowiecki, N., Weingartner, E., Baltensperger, U. and Buchmann, B., 2007. Contribution of railway traffic to local PM10 concentrations in Switzerland. Atmospheric Environment, 41(5), pp.923-933.

[6] Salma, I., Weidinger, T. and Maenhaut, W., 2007. Time-resolved mass concentration, composition and sources of aerosol particles in a metropolitan underground railway station. Atmospheric Environment, 41(37), pp.8391-8405.

[7] Barrero, R. , Tackoen, $X$ and van Mierlo, J., Stationary or onboard energy storage systems for energy consumption reduction in a metro network Proceedings of the Institution of Mechanical Engineers, Part F: Journal of Rail and Rapid Transit, vol. 224, pp. 207-225, 2010.

[8] Ceraolo, M. and Lutzemberger, G., Stationary and on-board storage systems to enhance energy and cost efficiency of tramways, Journal of Power Sources, vol. 264, pp. 128-139, ISSN 0378-7753, http://dx.doi.org/10.1016/j.jpowsour.2014.04.070, 2014.

[9] Gonzalez-Gil, A., Palacin, R. and Batty, P., Sustainable urban rail systems: Strategies and technologies for optimal management of regenerative braking energy, Energy Conversion and Management, vol. 75, pp. 374-388, ISSN 0196-8904, http://dx.doi.org/10.1016/j.enconman.2013.06.039, 2013.

[10] Gonzalez-Gil, A., Palacin, R., Batty, P. and Powell, J.P., A systems approach to reduce urban rail energy consumption, Energy Conversion and Management, vol. 80, pp. 509-524, ISSN 0196-8904, http://dx.doi.org/10.1016/j.enconman.2014.01.060, 2014.

[11] Barrero, R., Van Mierlo, J. and Tackoen, X., Energy savings in public transport, Vehicular Technology Magazine, IEEE, vol.3, no.3, pp.26,36, doi: 10.1109/MVT.2008.927485, 2008.

[12] Steiner, M., Klohr, M. and Pagiela, S., Energy storage system with ultracaps on board of railway vehicles, Power Electronics and Applications, European Conference, pp.1-10, doi: 10.1109/EPE.2007.4417400, 2007.

[13] Thiounn-Guermeur, M., Evaluation of the hybrid locomotive PLATHEEA Platform for Energy Efficiency and Environmentally Friendly Hybrid Trains, Proceding of WCRR (World Congress of Railway Research), France, 2011.

[14] Pugi L., Conti R., Nocciolini D., Galardi E., Meli E. (2015). A Comprehensive Tool for the Optimization of Traction and Braking systems with respect to the Application of Energy Storage devices. INTERNATIONAL JOURNAL OF RAILWAY TECHNOLOGY, vol. 4, p. 69-93, ISSN: 2049-5358, doi: 10.4203/ijrt.4.1.4

[15] R. Conti, E. Galardi, E. Meli, D. Nocciolini, L. Pugi, A. Rindi (2015). Energy and wear optimisation of train longitudinal dynamics and of traction and braking systems. VEHICLE SYSTEM DYNAMICS, p. 122, ISSN: 0042-3114, doi: 10.1080/00423114.2014.990466

[16] Ramakrishnan, R., Hiremath, S.S. and Singaperumal, M., 2014. Dynamic Analysis and Design Otptimization of Series Hydraulic Hybrid System through Power Bond Graph Approach. International Journal of Vehicular Technology, 2014.

[17] Allotta Pugi - Meccatronica: Elementi di Trazione Elettrica, Esculapio Editore I Ed. 2013 Vol. form.to $17 \times 24$ in brossura Pag. 268 ISBN: 9788874885855 DOI: $10.15651 / 978-88-748-8585-5$

[18] Muller R., Veranderungen von Radlaufflachen im Betriebseinsatz das Fahrzeugverhalten (Teil 1) ZEV+DET Glasers Annalen, Vol 588.

[19] F. Perticaroli SISTEMI ELETTRICI PER I TRASPORTI Isbn 9788808-08495-8 2001 Ed. Ambrosiana

[20] Dario Zaninelli, Sistemi elettrici per l'alta velocità ferroviaria ISBN 97888-7398-069-4 Polipress Ed. 2010. 